\title{
Micrometastases: are they clinically significant disease?
}

O'Sullivan and colleagues (page 512) argue that micrometastases in the bone marrow are common in patients who have carcinoma of the oesophagus, stomach and colorectum. Both before and six months or more after surgery, they believe these micrometastases can be detected in a quarter of patients with colorectal cancers and one third of patients with gastric cancers. Micrometastases were also found after surgery in about half of the patients with squamous oesophageal cancer. The presence of these cells in the bone marrow preoperatively was of no clinical importance. But half the patients with bone marrow micrometastases detected postoperatively relapsed within 18 months. In contrast, less than one fifth without bone marrow abnormalities developed recurrent disease. Their paper, up to this point, agrees with numerous publications which have similarly shown that the presence of tumour cells within the bone marrow in cancers of the breast, lung, prostate, colon, and stomach is associated with a worse prognosis. However, O'Sullivan et al go one step further and conclude that the presence of postoperative micrometastases indicates minimal residual disease.

Studies such as this one are important. More appropriate use of adjuvant chemotherapy is one way to improve the outcome of patients with gastrointestinal cancer and simple, accurate methods to identify patients at risk are needed. The detection of micrometastases in the bone marrow, however, is not foolproof.

This (and other) studies have a false positive rate in controls of about $2 \%$. In addition, more than half the patients in whom abnormalities were detected did not relapse after a maximum of 18 months' follow up. It has been suggested that the epithelial antigen targeted here may not be sufficiently specific ${ }^{1}$; perhaps screening using PCR or RT-PCR technology for more certain markers such as $\mathrm{H}$ - or $\mathrm{Ki}$-ras mutations or microsatellite instability may improve the accuracy rate. Such techniques now seem to be sufficiently sensitive to identify circulating tumour cells in plasma samples. ${ }^{2} 3$

With oesophageal cancers, no adjuvant chemotherapy has yet been shown to be beneficial. Meta-analysis of studies in patients with gastric carcinomas shows only a small benefit from additional treatment. However, in both these diseases optimal studies are still awaited. It is interesting that none of the patients in the study by O'Sullivan et al were apparently considered for adjuvant treatment. In contrast, randomised clinical trials have proved that patients with resected colorectal cancer at high risk of recurrence can benefit from adjuvant treatment, although in the UK, fewer than half of these patients are referred for an opinion about such treatment. ${ }^{4}$

Before these authors' findings could contribute to clinical practice, several questions need to be answered. The first is a practical one. Appropriate adjuvant chemotherapy for colorectal cancer has increased the cure rate by up to $15 \%$. This is not a trivial figure, particularly considering how frequently this diagnosis is made. (By way of comparison, the use of thrombolysis for myocardial infarction only prevents death in some $2 \%$ of patients. Cost benefit analysis also shows that adjuvant chemotherapy for colorectal cancer is considerably cheaper per life saved than thrombolysis. ${ }^{5}$ ) In addition, adjuvant chemotherapy for colorectal carcinoma also improves life expectancy even if the patient does relapse subsequently. However, the studies which have shown benefit from adjuvant therapy have relied on early treatment (within three months of surgery) and the trend is for ever earlier adjuvant chemotherapy, even on the first postoperative day in a large study running in the USA. Therefore, O'Sullivan and colleagues' method of predicting risk by examining the bone marrow six months postoperatively may well provide information, but are they not trying to close the stable door long after the horse has bolted?

So, to start with, we need to know precisely when after surgery abnormalities detected in the bone marrow are important. We need to know how many cells should be obtained per aspirate and how many sites should be aspirated. We also need to know how many abnormal cells within the aspirate are significant. Others have shown that less than three abnormal cells in an aspirate of $10^{6}$ bone marrow cells in patients with gastric cancer ${ }^{6}$ and less than 10 in patients with breast cancer ${ }^{7}$ are of no practical consequence.

Another problem confronting their theory that these cells indicate residual disease is the way these carcinomas recur. Even in breast cancer where bone metastases are often seen, bone marrow micrometastases are more commonly associated with relapse in sites away from the bone than in the bone itself. $^{8}$ However, in gastric and colorectal cancer bone metastases are unusual, even in very advanced disease, which is difficult to reconcile with the concept that these abnormal cells constitute clusters of residual tumour. If there is residual disease as the authors believe, why does it not become clinically relevant? Could it rather be that the immunocytochemistry techniques are picking up non-viable or dormant cells? Probably not, as the balance of evidence suggests that the presence of these abnormal cells in bone marrow simply reflects the high metastatic potential of primary and recurrent cancers rather than established microscopical deposits of residual disease. ${ }^{9}$

Our final caveat concerns the issue of staging. O'Sullivan and colleagues' initial staging of their patients was indeed "careful" and far in excess of conventional practice, at least in the UK, but even so it may not have been adequate. Chest radiography is not sufficiently sensitive to diagnose the presence of (potentially resectable) lung metastases which are best detected on thin slice computed tomography scanning. Only one of their patients relapsed in the chest, but this lesion may have been visible at the time of surgery or when the postoperative bone marrow aspirate was taken if a scan had been performed.

This study describes a complicated procedure which can pick up tumour cells as they travel through the bone 
marrow. Undoubtedly, it has a role as a research tool. However, it will be the technology which enables discovery of tumour cells in the peripheral blood which is likeliest to become relevant to clinical practice in the future.

H J N ANDREYEV

Department of Medicine

D CUNNINGHAM

The Gastrointestinal Unit,

Royal Marsden Hospital and the Centre for Cancer Therapeutics,

The Institute of Cancer Research,

Downs Road, Sutton, Surrey SM2 5PT

1 Traweek ST, Liu J, Battifora $\mathrm{H}$. Keratin gene expression in non-epithelia tissues. Am $\mathcal{F}$ Pathol 1993; 142: 1111-8.
2 Chen XQ, Stroun M, Magnenat J-L, et al. Microsatellite alterations in plasma DNA of small cell lung cancer patients. Nat Med 1996; 2: 033-5.

3 Nawroz H, Koch W, Anker P, Stroun M, Sidransky D. Microsatellite alterations in serum DNA of head and neck cancer patients. Nat Med 1996; 2: 1036-7.

4 Kerr DJ, Gray R. Adjuvant chemotherapy for colorectal cancer. $\mathrm{Br} \mathcal{f}$ Hosp Med 1996; 55: 259-62.

5 Brown ML, Nayfield SG, Shibley LM. Adjuvant therapy for stage III colon cancer: economic returns to research and cost effectiveness of treatment. f Natl Cancer Inst 1994; 86: 424-30.

6 Goldman L. Cost and quality of life: thrombolysis and primary angioplasty. f Am Coll Cardiol 1995; 25 (suppl): 38S-41S

7 Jauch K-W, Heiss MM, Gruetzner U, et al. Prognostic significance of bone marrow micrometastases in patients with gastric cancer. $\mathcal{f}$ Clin Oncol 1996; 14: 1810-7.

Cote RJ, Rosen PP, Lesser ML, Old LJ, Osborne MP. Prediction of early
relapse in patients with operative breast cancer by detection of occult bone marrow micrometastases. $\mathcal{F}$ Clin Oncol 1991; 9: 1749-56.

9 Mansi JL, Easton D, Berger U, et al. Bone marrow micrometastases in primary breast cancer: prognostic significance after 6 years follow up. Eur $\mathcal{f}$ Cancer 1991; 27: 1552-5. 